\title{
Staged approach for the management of atrial septal defect in the presence of a small left ventricle and suprasystemic pulmonary pressure
}

\author{
Roie Tal MD, Yitzhack Schwartz MD, Avraham Lorber MD
}

R Tal, Y Schwartz, A Lorber. Staged approach for the management of atrial septal defect in the presence of a small left ventricle and suprasystemic pulmonary pressure. Curr Res Cardiol 2014;1(2): 114-116.

A 29-year-old woman presented with a symptomatic large atrial septal defect, a small left ventricle with a modest left atrium, mild to moderate nonrheumatic mitral valve incompetence, an apex-forming very large right ventricle and suprasystemic pulmonary artery pressure. Following

Dercutaneous closure of ostium secundum atrial septal defect (ASD) is a safe and effective alternative to surgery (1). Complete occlusion of the defect is generally the desired procedural outcome. However, there are clinical and morphological circumstances in which acute complete occlusion may worsen hemodynamics because this atrial communication serves as a 'pop-off' valve (2). Such situations have been previously described in the literature, and include children and adults with pulmonary arterial hypertension as well as closure of an ASD in elderly patients (3). In these settings, the use of a fenestrated occluder device was demonstrated to be beneficial (3). We present a case involving a young woman with an interesting cardiac morphology of a small left ventricle and suprasystemic pulmonary artery pressure. A staged approach, comprised of medical therapy followed by fenestrated device implantation, enabled gradual occlusion of the interatrial communication, leading to clinical and hemodynamic improvement.

\section{CASE PRESENTATION}

A previously healthy 29-year-old woman, who was diagnosed with an ASD when she was 14 years of age, became breathless on minor physical activity during pregnancy and remained decapacitated six months after spontaneous vaginal delivery of a healthy baby. She was referred to the authors as a tourist after several well-known cardiac and cardiothoracic services abroad elected not to intervene because of her unfavourable condition.

On echocardiography, her heart morphology revealed a very small left ventricle (LV) - left ventricular end diastolic diameter of only $26 \mathrm{~mm}$ (Figure 1A) - with mild to moderate nonrheumatic mitral valve regurgitation, a very large right ventricle (RV) and a $22 \mathrm{~mm}$ secundum ASD (Figure 1B), with reasonable rims morphologically amenable to percutaneous device occlusion. The shunt was mainly left to right; however, a small, narrow, systolic jet of tricuspid valve incompetence crossed the ASD from right to left. The tricuspid valve regurgitation jet velocity was $5 \mathrm{~m} / \mathrm{s}$, suggestive of systolic pulmonary artery pressure (PAp) of approximately $120 \mathrm{mmHg}$, while the systemic pressure was $95 / 65 \mathrm{mmHg}$. A very small pericardial effusion was also noted.

\begin{abstract}
one year of preprocedural drug treatment to lower pulmonary hypertension, she underwent percutaneous closure of the atrial septal defect with a customized fenestrated device that enabled gradual adaptation to the occlusion of the interatrial communication. This special case demonstrates the benefits of using a fenestrated occluder device in patients with pulmonary hypertension and components of left ventricular diastolic dysfunction, which is considered to be high risk and not amenable to therapeutic intervention.
\end{abstract}

Key Words: Atrial septal defect; Fenestrated device; Percutaneous closure; Pulmonary hypertension

With regard to the possible etiology of increased PAp, there were no clinical or laboratory features of pulmonary embolism or a systemic illness such as Sjögren syndrome or lupus erythematosus, and the thrombophilia workup was negative. The patient was started on a combined drug therapy of bosentan $125 \mathrm{mg}$ twice/day, sildenanfil $20 \mathrm{mg}$ three times/day, bisoprolol $2.5 \mathrm{mg} /$ day and furosemide $20 \mathrm{mg} /$ day. The addition of bosentan aimed to decrease pulmonary resistance, thus decreasing the right-to-left shunt caused by the tricuspid valve jet through the ASD. Marked clinical and symptomatic improvements were documented soon thereafter and she returned home.

Being a tourist, the patient could return for a follow-up visit only one year after the initial evaluation. Following one year of treatment, her $\mathrm{O}_{2}$ saturation on room air increased from $88 \%$ to $90 \%$ to $98 \%$, and her subjective limited walking capability improved. Doppler interrogation of tricuspid valve regurgitation revealed a pressure gradient of $60 \mathrm{mmHg}$. After informed consent was obtained, a hemodynamic study was performed in the catheterization laboratory. The measured PAp was 78/28 mmHg (mean $44 \mathrm{mmHg}$ ); the pulmonary capillary wedge pressure was $12 / 7 \mathrm{mmHg}$ (mean $10 \mathrm{mmHg}$ ); and the Qp:Qs ratio was 3:1. The interatrial septum measured $50 \mathrm{~mm}$. The ASD stretched diameter was $24 \mathrm{~mm}$, with a small inferior fenestration revealed by transesophageal echocardiography. Although the LV was small, its systolic function was good. There was a large, apex-forming RV with good function. A sizing balloon was inflated through the ASD, resulting in an increase in mean pulmonary capillary wedge pressure from $10 \mathrm{mmHg}$ to $12 \mathrm{mmHg}$.

A decision was made to use a prefabricated $24 \mathrm{~mm}$ occluder device (Occlutech Figulla Flex ASD; H + H Maslanka GmbH, Germany), with a $7 \mathrm{~mm}$ fenestration that was customized and designed for this patient. The fabric of the device was cut to create the fenestration, offering a short-term potential vent mechanism in view of the small LV and modest left atrium. However, the nitinol mesh was left intact to allow for future endothelial proliferation and gradual closure of the fenestration (Figure 2). The prospect of spontaneous eventual closure of the fenestration at a later stage, obviating an additional intervention, was naturally sought. Immediately after the successful deployment of the closure device, the magnitude of the mitral valve

Pediatric Cardiology and Adults with Congenital Heart Disease, Rambam Health Care Campus, Haifa, Israel

Correspondence: Dr Avraham Lorber, Pediatric Cardiology and Adults with Congenital Heart Disease, Rambam Health Care Campus, Meyer Children's

Hospital, Haifa, Israel. Telephone 972-4-7772275, fax 972-4-7772175, e-mail a_lorber@rambam.health.gov.il 


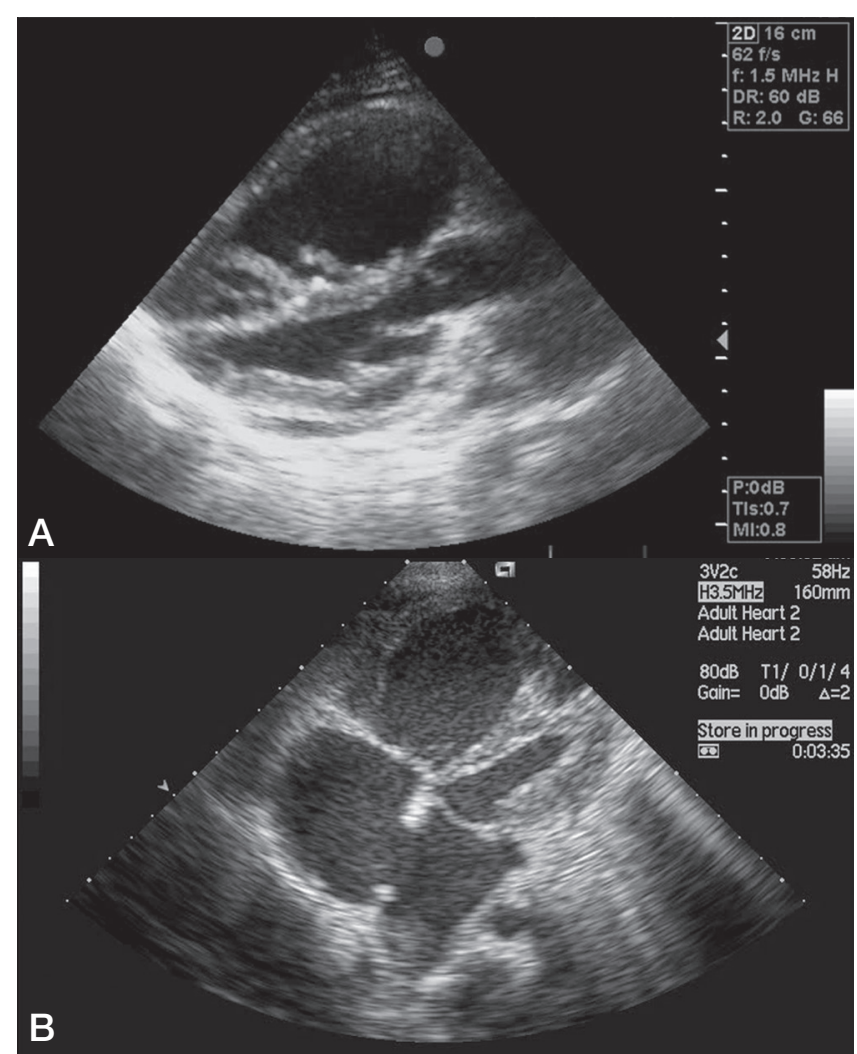

Figure 1) A Transthoracic echocardiogram in long-axis view demonstrating a large right ventricle and a small left ventricle with minor posterior pericardial effusion. B Transthoracic echocardiogram in four-chamber view demonstrating a large right ventricle, a small left ventricle and a large secundum atrial septal defect with rims suitable for transcatheter device closure

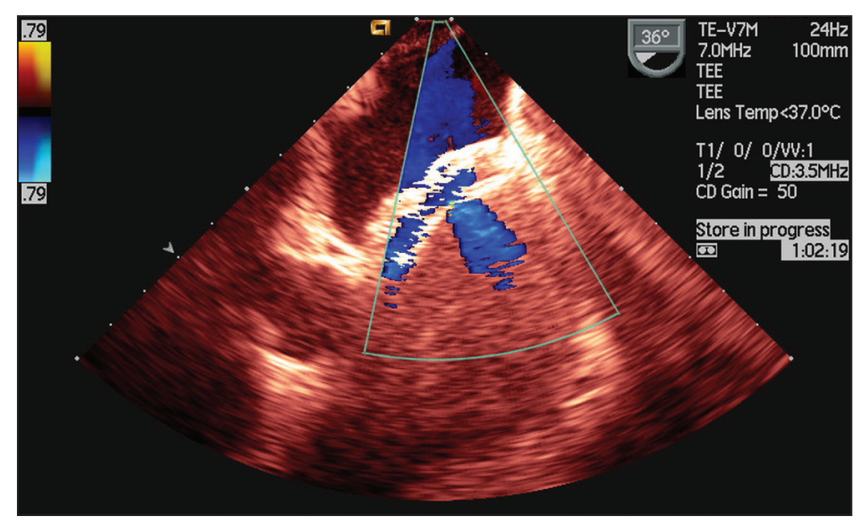

Figure 2) Intraprocedural transesophageal echocardiography illustrating the atrial septal defect occluder in situ. A left-to-right shunt is seen (in blue) across the $7 \mathrm{~mm}$ fenestration. There is also flow between the discs - immediately following device release

regurgitation increased modestly from $1 / 4$ to $2 / 4$. In addition to bosentan and sildenanfil, acetylsalicylic acid $100 \mathrm{mg} /$ day and ramipril $2.5 \mathrm{mg} /$ day were given. On transthoracic echocardiogram (TTE) the day after the procedure, the LV was already slightly increased in size, and the Qp:Qs ratio was 1.5:1.

On clinical and TTE evaluation three months after the procedure, the patient was asymptomatic, with a tricuspid valve regurgitation flow velocity of $3 \mathrm{~m} / \mathrm{s}$ and a systolic PAp estimated to be $45 \mathrm{mmHg}$. The LV increased up to $40 \mathrm{~mm}$ in size in end diastole, with normal function.

The patient returned for a follow-up two years after the procedure. Her physical examination was normal except for a soft, mid-systolic

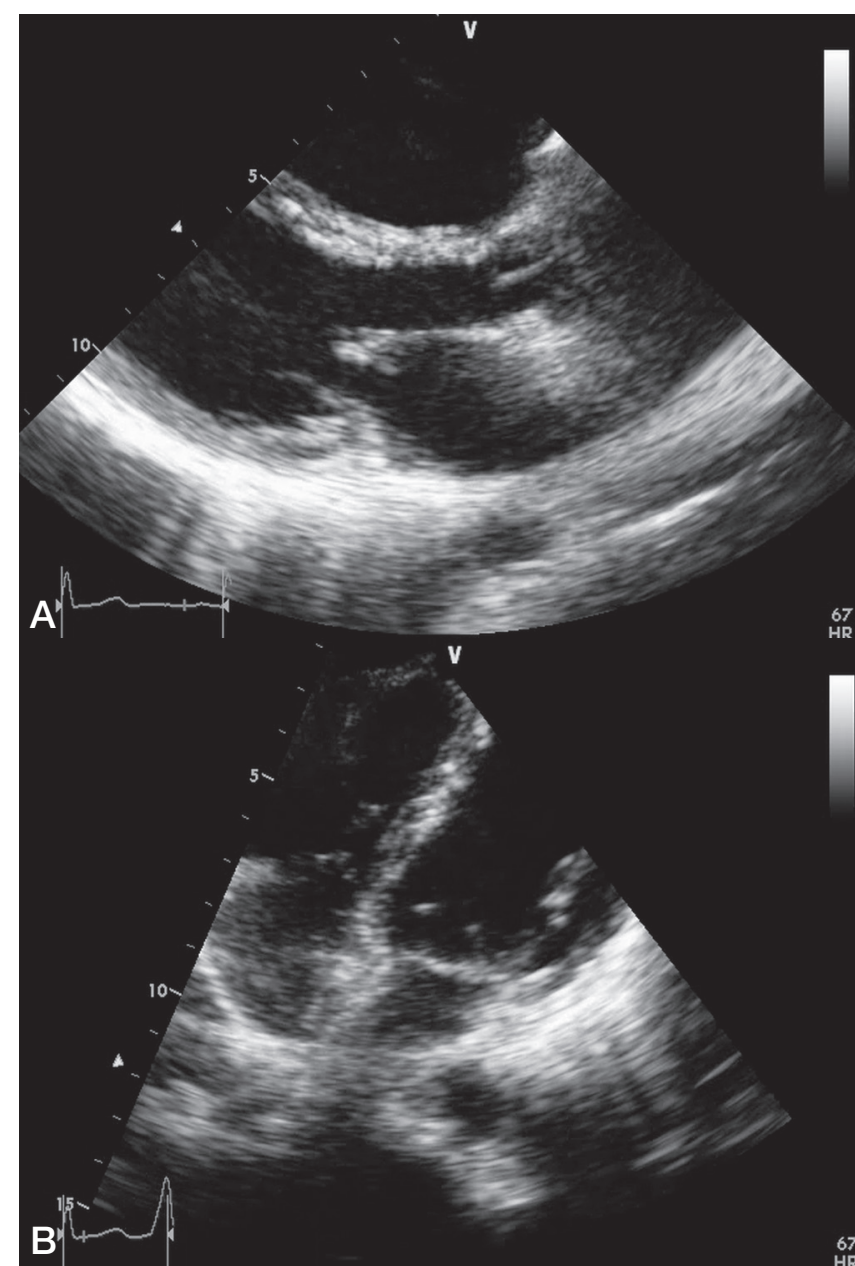

Figure 3) A Transthoracic echocardiogram in long-axis view two years after the procedure demonstrating normal left ventricle dimensions. B Transthoracic echocardiogram in four-chamber apical view (with slight apical foreshortening) two years after the procedure demonstrating reasonable left ventricle size and an optimal position of the atrial septal defect occluder

heart murmur. TTE demonstrated normal left heart chamber size (Figure 3A), normal ventricular function with no hypertrophy and no significant residual RV enlargement. The mitral regurgitation improved due to LV remodelling and became very mild. The configuration, motion and Doppler flows of the pulmonary, tricuspid and aortic valves were normal, with trivial physiological tricuspid regurgitation, with a systolic flow velocity of $2.7 \mathrm{~m} / \mathrm{s}$, compatible with a Doppler gradient of $29 \mathrm{mmHg}$, representing an upper normal or borderline elevation of PAp estimated to be $35 \mathrm{mmHg}$. The deployed closure device was optimally positioned with no residual shunt or interference with heart valve function (Figure 3B).

\section{DISCUSSION}

ASD accounts for $10 \%$ to $17 \%$ of all congenital cardiac malformations (4). It should be diagnosed and closed preferably during childhood. However, because the majority of children with ASD are asymptomatic, a substantial number of patients may present as symptomatic adults. Untreated ASD in adults leads to further RV dilation and failure due to progressive volume overload, and later pulmonary hypertension, which causes RV hypertrophy due to pressure overload (5). Other complications of untreated ASD in adults may include arrhythmia (mostly atrial fibrillation), paradoxical emboli and stroke.

Ewert et al (6) reported on the potential complications of percutaneous closure of ASDs in elderly patients. They examined the hemodynamic changes that occurred after temporary balloon occlusion of 
the interatrial septum in 18 patients $>60$ years of age, seven of whom showed a marked increase in left atrial pressure. Restrictive physiology of the LV is more common in elderly patients than in children. In the study by Ewert et al (6), a patient who underwent closure with an ASD device developed pulmonary edema that required mechanical ventilation. The authors also questioned whether such a combination should be considered a contraindication for ASD closure.

Further studies have recommended preconditioning of the LV before ASD closure in adults $>60$ years of age (6-9). The option of partial closure of the defect by deployment of a fenestrated device has also been presented (10). Such devices were either preordered, custommade fenestrated devices or regular septal occluders with manually created fenestrations. Data are scarce regarding this technique in children or young adults. Kenny et al (11) reported a 46-year-old patient with ASD and left ventricular diastolic dysfunction due to hypertension, which was successfully treated with a fenestrated septal occluder (Helex, Gore Medical, USA).

Another group of patients that may require partial closure of an existing ASD are those with concurrent pulmonary hypertension. Older patients with an ASD and pulmonary hypertension may need the fenestration as a protective means for decompression of the RV in case of a pulmonary hypertensive crisis (10). A balloon occlusion test should be performed for ASD occlusion tolerance and to enable hemodynamic evaluation before ASD closure (10).

Other patients who may need evaluation by balloon occlusion of ASD before closure include those with ischemic coronary disease and dilated cardiomyopathy (12).

Unless sutures are placed in the nitinol mesh after creating the fenestration, the memory properties of the nitinol will reshape the device to its original configuration, thus closing the fenestration after the acute recovery phase (13). Potential risks for residual shunt through a fenestrated device are infective endocarditis and cerebrovascular events caused by paradoxical emboli, septic emboli or local formation of thrombi. Therefore, the eventual complete closure of the fenestration may be desirable (13). This could be achieved by a fenestration small enough to close spontaneously by endothelial ingrowth or by implanting a second device for the closure of the remaining shunt at a later stage.

Our patient presented with several interrelated problems. There was a large ostium secundum ASD, a small LV with a modest left atrium, mild to moderate nonrheumatic mitral valve incompetence,

\section{REFERENCES}

1. Du ZD, Hijazi ZM, Kleinman CS, Silverman NH, Larntz K. Aplatzer Investigators. Comparison between transcatheter and surgical closure of secundum atrial septal defect in children and adults: Results of a multicenter nonrandomized trial. J Am Coll Cardiol 2002; 39:1836-44.

2. Skinner GJ, Tulloh RM, Tometzki AJ, Schulze-Neick I, Morgan GJ. Atrial septal defect closure with an Amplatzer septal occluder fenestrated with a coronary stent in a child with pulmonary arterial hypertension. Cardiol Young 2012;23:1-5.

3. Lammers AE, Derrick G, Haworth SG, Bonhoeffer P, Yates R. Efficacy and long-term patency of fenestrated Amplatzer devices in children. Catheter Cardiovasc Interv 2007;70:578-84.

4. Rigby ML. Atrial septal defect. In: Gatzoulis MA, Webb GD, Daubeney PE, eds. Diagnosis and Management of Adult Congenital Heart Disease, 1st edn. Spain: Churchill Livingstone; 2003:163-70.

5. Vogel M, Berger F, Kramer A, Alexi-Meshkishvili V, Lange PE. Incidence of secondary pulmonary hypertension in adults with atrial septal or sinus venosus defects. Heart 1999;82:30-3.

6. Ewert P, Berger F, Nagdyman N, et al. Masked left ventricular restriction in elderly patients with atrial septal defects: A contraindication for closure? Catheter Cardiovasc Interv 2001;52:177-80.

7. Peters B, Ewert P, Schubert S, et al. Self-fabricated fenestrated Amplatzer occluders for transcatheter closure of atrial septal defect an apex-forming very large RV and marked suprasystemic PAp. Each of these components had to be managed simultaneously because dismissing any of them carried the risk for heart failure. The low $\mathrm{O}_{2}$ saturation on room air and exertional dyspnea improved with medical therapy. The pulmonary pressures also gradually decreased with medical therapy and, following the interventional procedure, there was further reduction in pressure from suprasystemic to upper normal levels. The timing of the medical and interventional therapy was dependent on the availability of the patient, who is a foreign resident.

In view of the elevated PAp and small LV, a fenestrated device was fabricated. A single, large fenestration $7 \mathrm{~mm}$ in diameter was preferred over multiple smaller fenestrations to benefit from a longer period of venting mechanism, and to avoid premature complete occlusion by endothelization. It was assumed that the residual shunt would be of minor dimensions and a have a modest hemodynamic effect. However, it may have a significant role in the early postprocedural stage, allowing a temporary vent mechanism and avoiding left atrial pressure overload in the presence of a small LV. Using a custom-made device cutting out the fenestration through the three layers of the fabric without interfering with the original metal mesh of the device - has, in fact, enabled effective short-term protection and gradual adaptation and eventual long-term spontaneous full closure by the ingrowth of endocardium, as expected.

The overall positive outcome of the patient was very reassuring. There was no residual shunt through the device. The LV increased in size, reaching normal dimensions. The RV, although still slightly enlarged, had good function with normalization of PAp.

\section{CONCLUSION}

Proper preprocedural conditioning and the prudent implantation of a fenestrated occluder device enabled successful, gradual closure of a large ASD in a patient who was at risk for cardiac failure.

ACKNOWLEDGEMENT: The patient provided informed consent. All authors participated in the research, and have reviewed and agree with the content of the article.

DISCLOSURES: The authors have no financial disclosures or conflicts of interest to declare.

in patients with left ventricular restriction: Midterm results. Clin Res Cardiol 2006;95:88-92.

8. Holzer R, Cao QL, Hijazi ZM. Closure of a moderately large atrial septal defect with a self-fabricated fenestrated Amplatzer septal occluder in an 85-year-old patient with reduced diastolic elasticity of the left ventricle. Catheter Cardiovasc Interv 2005;64:513-8.

9. Schubert S, Peters B, Abdul-Khaliq H, Nagdyman N, Lange PE, Ewert P. Left ventricular conditioning in the elderly patient to prevent congestive heart failure after transcatheter closure of atrial septal defect. Catheter Cardiovasc Interv 2005;64:333-7.

10. Kretschmar O, Sglimbea A, Corti R, Knirsch W. Shunt reduction with a fenestrated Amplatzer device. Catheter Cardiovasc Interv 2010;76:564-71.

11. Kenny D, Cao QL, Hijazi ZM. Fenestration of a Gore Helex Septal Occluder device in a patient with diastolic dysfunction of the left ventricle. Catheter Cardiovasc Interv 2011;78:594-8.

12. Cheatham JP. Now we are making a hole in a device meant to close a hole: Why? How? Is there a better answer? Catheter Cardiovasc Interv 2005;64:519-21.

13. Schneider HE, Jux C, Kriebel T, Paul T. Fate of a modified fenestration of atrial septal occluder device after transcatheter closure of atrial septal defects in elderly patients. J Interv Cardiol 2011;24:485-90. 